\title{
Reading and understanding an antibiogram
}

\author{
Carlo Tascini, ${ }^{1}$ Emanuela Sozio, ${ }^{2}$ Bruno Viaggi, ${ }^{3}$ Simone Meini ${ }^{4}$ \\ ${ }^{1}$ First Division of Infectious Diseases, Cotugno Hospital, Azienda Ospedaliera dei Colli, Napoli; ${ }^{2}$ Department of Emergency \\ Medicine, University-Hospital of Pisa; ${ }^{3}$ Department of Neuroanesthesia and Intensive Care, Careggi University-Hospital, \\ Firenze; ${ }^{4}$ Department of Internal Medicine, S.M. Annunziata Hospital, Firenze, Italy
}

\begin{abstract}
In recent years, we have been facing a significant increase in antimicrobial resistance and complex enzymatic mechanisms. The challenge of antibiotic resistance is becoming dramatic. Among gram-positive it is spreading resistance to glycopeptides, reducing the possibility to use these drugs empirically. Among gram-negative rods, beside the spreading of extended-spectrum $\beta$-lactamases, there is an increased diffusion of carbapenemases. In order to administer the correct antibiotic therapy, physicians need a rapid and correct interpretation with non-automated tests to implement appropriate therapeutic strategies. The automated reporting systems do not always provide complete and accurate information on antimicrobial resistance phenotype, making it difficult to interpret. Recently, the European Committee of Antimicrobial Susceptibility Testing (EUCAST) has proposed a new breakpoint system to be adopted by European countries. An interpretative reading of an antibiogram aims to analyze the overall susceptibility pattern, not just the result for an individual antibiotic, and so to predict the underlying resistance mechanisms. The purpose of this work is to guide physicians in reading and understanding antibiograms through an attempt of phenotypic interpretation of resistance mechanism.
\end{abstract}

\section{Introduction}

In recent years, we have been observing a significant increase in antimicrobial resistance with multiple and often combined enzymatic mechanisms affecting an increasing number of microorganisms.

In the past, interpretative reading of the antibiogram was used to infer resistance mechanisms behind resistant phenotypes, to identify organism for infection control purpose and to apply expert rules. Automated interpretative reading can never represent a complete strategy as identifying resistance mechanisms by genetic and biochemical investigations. In fact, bacteria with multiple resistance determinants (affecting the same class or classes of antibiotics) are increasingly fre-

Correspondence: Carlo Tascini, I Divisione Malattie Infettive, Ospedale D. Cotugno, via Quagliariello 54, 80131 Napoli, Italy.

Tel.: +39.081.7067282 - Fax: +39.081.7067426.

E-mail: carlo.tascini@libero.it

Key words: Antibiogram; susceptibility test; minimal inhibitory concentration; antibiotic.

See online Appendix for supplementary tables.

Received for publication: 6 October 2016.

Accepted for publication: 20 October 2016.

This work is licensed under a Creative Commons Attribution NonCommercial 4.0 License (CC BY-NC 4.0).

CCopyright C. Tascini et al., 2016

Licensee PAGEPress, Italy

Italian Journal of Medicine 2016; 10:289-300

doi:10.4081/itjm.2016.794 quent and the resistance patterns with multiple mechanisms may be confusing or misleading. Moreover, interpretative reading cannot identify new resistance mechanisms if these give a resistance profile identical to that given by a known mechanism. Furthermore, some species often have complex multi-resistance profiles that are difficult to relate reliably to a geneticallydefined mechanism. Therefore, the only method able to define precisely the mechanisms of resistance is the genotypic one, but in clinical practice is difficult to apply and may have several limitations.

An interpretative reading of an antibiogram by clinicians aims to analyze the overall susceptibility pattern, not just the result for an individual antibiotic, and so to predict the likely underlying resistance mechanisms. In this context, susceptibilities that appear questionable should be identified and retested, and the possible usefulness of drugs should be further tested.

Clinicians should know these interpretative rules and update their knowledge continuously.

The purpose of this work is to guide physicians in reading and understanding antibiograms through an attempt of phenotypic interpretation of resistance mechanism exhibited by the microorganisms that are isolate in clinical practice.

\section{Part I: General considerations}

\section{Susceptibility tests}

The antibiogram is the result of susceptibility tests in which the microorganism is in vitro essayed for its susceptibility to several antibiotics tested in different concentrations. The main tests are: Kirby Bauer, broth micro-dilution (BMD) and gradient method (E-test). 
Kirby-Bauer disk diffusion method provides for the evaluation of the diameters of the inhibition zones around the disks containing the antibiotic tested; more frequently BMD (method that can be automated) is used, and represents the reference method. Currently, to provide faster reporting of susceptibility results and to face the large number of tests that come in centralized laboratories, they use automated systems, such as Vitek2 (bioMérieux Italia S.P.A., Grassina (FI), Italy).

\section{Minimum inhibitory concentration}

BMD and automated systems allow obtaining the minimum inhibitory concentration (MIC), which is the lowest concentration of antibiotic that is able to inhibit the in vitro growth of the microorganism after 18-24 h of incubation. E-test is a manual method, performed in agar, using a paper strip with a gradient concentration of a given antibiotic that is able to obtain MICs and is useful as a supplement to the other methods. KirbyBauer does not provide MIC values, but diameters of inhibition zones.

The diameters of inhibition zones and MICs must be compared to clinical breakpoints standardized for different organism-antibiotic combinations. Breakpoints are established by specific committees, one in Europe [European Committee on Antimicrobial Susceptibility Testing (EUCAST)] and one in the USA [U.S. Clinical and Laboratory Standards Institute (CLSI)].

An interpretative reading on an antibiogram requires that isolates are identified accurately to species level and tested with an adequate number of appropriate antibiotics. Antibiograms should be read with attention to recognize unusual results and drugs best prevented owing to their risk of selecting resistance and using indicator drugs. An indicator drug is used to detect the presence of resistance also to the related agents and it is chosen as the member of the drug family to which the mechanism gives the most obvious resistance.

\section{European Committee on Antimicrobial Susceptibility Testing: what is it?}

In 1997 EUCAST has unified the different standards for interpreting the antibiogram previously used in six European countries. The EUCAST is a committee jointly organized by the European Society for Clinical Microbiology and Infectious Diseases (ESCMID), by the European Centre for Disease Prevention and Control (ECDC) and by the six national committees previously active. Up to now, breakpoints defined by EUCAST are the only officially recognized by the European Medicines Agency (EMA), body authorizing the placing on the market of drugs in the countries of the European Union.

In EUCAST pharmaceutical companies have func- tion of consultation but have no decision-making power in setting breakpoints.

Italian microbiological laboratories have adopted EUCAST Guidelines since 2011 (previously CLSI standards were adopted). ${ }^{1,2}$ EUCAST documents are free and consultable on the web at http://www.eucast.org.

\section{Clinical breakpoints and clinical categories}

Breakpoints are set according to several parameters: microbiological, pharmacological (relationship between $\mathrm{PK} / \mathrm{PD}$ index and response to treatment) and clinical (best evidence from literature). They are ultimately derived from human clinical studies comparing outcomes with the MICs for the infecting pathogen.

Breakpoints are used to define the clinical category of susceptibility: susceptible (when bacterial strain growth is inhibited in vitro by a concentration of the antibiotic that is associated with a high likelihood of therapeutic success), intermediate (uncertain probability of successful treatment), resistant (high likelihood of therapeutic failure).

For each organism-antibiotic combination are set two breakpoints (in this case there are 3 categories of interpretation: susceptible, intermediate, resistant) or just a breakpoint (so providing for two categories of interpretation: susceptible and resistant).

The aim of clinical breakpoints is to use MIC values to separate strains where there is a high likelihood of treatment success using in vivo the given antibiotic from those whose treatment is more likely to fail due to a resistance mechanism. This is why we talk about clinical breakpoint (BC).

EUCAST breakpoints are often lower than those of the CLSI, ${ }^{1,2}$ with more restrictive interpretation of susceptibility, except for those related to carbapenems and Enterobacteriaceae. The EUCAST breakpoints are available, both for Kirby-Bauer disk diffusion method and micro-dilution broth test, and free on the web at http://www.eucast.org.

Laboratories and clinicians should be conscious of the natural resistance phenotypes of common pathogens. Natural resistances are shown in Appendix. In some cases, a report of susceptible or resistant is unexpected: if unusual resistance or susceptibility pattern is found it is reasonable to be skeptical. New resistances of public health concern should be recognized. When a resistance emerges by high frequency mutation there is the risk to be selected in the patient during therapy.

\section{Epidemiological cut-off}

EUCAST, differently from the CLSI, has defined the epidemiological cut-off (ECOFF), ${ }^{1}$ which is the concentration value that divides wild type isolates (that do not have any acquired and mutational resistance mechanisms) from the population of strains that have 
resistance mechanisms. The epidemiological breakpoints are important in the choice of the antibiotic and the drug dosages.

Often in automated antibiograms MICs are reported $\leq$ at the minimum concentration tested regarding some antibiotics, but in some cases, for example for Enterobacteria and quinolones, it is important to know the accurate MIC. In those cases where the epidemiological cut-off is different from the clinical breakpoints: to know with accuracy the MIC allows to understand if the germ has already developed some mutation that could let mono-therapy to fail.

\section{Changes on interpretation of susceptibility and changing rules}

We must know that the reporting rules of the antibiograms are not immutable over time, but may change in relation for example to the need to put in place new strategies for limiting the spread of resistance or based on new evidence in the literature.

Since 2010, some changes were made in the interpretation of susceptibility, especially for Gram-negative rods.

In addition to lowering of the breakpoint for some specific microorganism-antibiotic combinations, some combinations will be deleted from reporting as they are considered inadequate in the therapeutic area, or the microorganism is naturally resistant to the drug, or because there is no clinical evidence.

Both, CLSI and EUCAST, decided in a separate process to modify breakpoints for oxyminocephalosporins and Enterobacteria. ${ }^{1,2}$

Moreover, the new rule recommended, as Enterobacteriaceae and cephalosporins or carbapenems, reporting MIC results as found, and do not search anymore the mechanism of resistance, such as extended-spectrum $\beta$-lactamases (ESBL) or carbapenemases, except for epidemiological purposes and for infection control. Then ESBL-producing enterobacteria could be reported as susceptible to some cephalosporins and resistant to another and the interpretation will be based on clinical breakpoints: it is no longer required to test for ESBL and consequently changes the result, as in the past.

\section{Interpretation of an antibiogram}

The interpretation of an antibiogram is much more than the categorization of susceptibility and represents an attempt of phenotypic interpretation of the resistance mechanisms exhibited by the microorganisms that are isolate in clinical practice.

The correlation between the data obtained in vitro (MIC or inhibition zone) and the real clinical efficacy of the drug depends on a complex set of factors, including the site of infection and the ability of that drug to reach the appropriate concentrations in the target site. Dose and effective drug delivery are obviously also important in terms of pharmacokinetics and pharmacodynamics.

The absolute value of the MICs reported on susceptibility testing must not be evaluated vertically between the different drugs tested. For example, if there is an antibiotic $X$ with a MIC of $0.5 \mathrm{mg} / \mathrm{L}$ and breakpoint 2 $\mathrm{mg} / \mathrm{L}$, and an antibiotic $\mathrm{Y}$ with a MIC of $2 \mathrm{mg} / \mathrm{L}$ but breakpoint of $16 \mathrm{mg} / \mathrm{L}$, the drug with a MIC more favorable is the antibiotic Y. This ratio is named MIC breakpoint quotient. Therefore, it should be useful to have the values of clinical breakpoints on the antibiograms.

Reading and understanding an antibiogram, and therefore its interpretation, is based on the recognition of the possible mechanism at the base of resistance, which can be extended to non-tested drugs or that lead the change of category obtained in vitro (as for example the presence of oxacillin-resistant Staphylococci determines resistance to all beta-lactams, except ceftaroline and ceftobiprole).

To properly use antibiotics, the characteristics of microbiological activity (susceptibility of the microorganism) should be considered as well as the pharmacokinetic and pharmacodynamics characteristics, even simply the penetration of the antibiotic into the site of infection.

For example, it is very important to know the bacteriostatic activity (antibiotics that only inhibit the growth) and bactericidal activity (antibiotics that kill): the mechanisms that allow the bacteriostasis can be different from those of the bactericidal either to the same drug that for the same bacterial species (penicillins are bactericidal against pneumococci but bacteriostatic against enterococci).

The clinician must also consider the potential to induce resistance in selecting the appropriate antibiotic therapy. For example mono-therapy with certain molecules can select resistance during the treatment: Staphylococci may develop resistance with mono-therapy with rifampicin or quinolones (these drugs should be considered as partners of another antibiotic); all strains of gram negative rods that are inducible AmpC chromosomal carriers such as Enterobacter can develop resistance to third-generation cephalosporins after induction and derepression of the enzyme; Enterobacteriaceae have the ability to select for resistant strains during mono-therapy with fosfomycin; Pseudomonas aeruginosa has the ability to develop resistance against all molecules of antibiotics except polymyxins.

Therefore, clinicians should keep themselves up to date on antibiotic resistance, to recognize the exceptional resistance phenotypes, natural resistance and combinations of antibiotic and organism for which there is high likelihood of resistance development to simple mutation. The clinician should be able to interpret the resistance phenotype in order to select the appropriate antibiotic therapy, or which has less impact on ecology 
of the system, causing less resistance, fewer adverse effects and cost whenever possible.

The basic concept is that you cannot think of reading an antibiogram if you do not previously know the main potential characteristics of resistance of the microorganism tested.

Table 1 shows exceptional resistance phenotypes, while Table 2 shows the bacteria that have the ability to develop resistance to a class of antibiotics, for single point mutation. ${ }^{3}$

\section{Antibiotic resistance}

Antibiotic resistance is a major limitation to the success of antibiotic therapy. The mechanism of resistance may be as varied as the modification of the site of action, the decreased permeability of the bacterial membrane, the expulsion of the drug through efflux pumps and inactivation of the drug.

A microorganism can have more than one mechanism of resistance, and such resistance can arise from one or several point mutations of the target genes or from the acquisition of new genes via plasmids or transposons. A mechanism of resistance can be functional (and always expressed) or inducible in the presence of a given antibiotic (these mechanisms might be difficult to detect in vitro).

If a resistance emerges by high frequency mutation, there is a significant risk that it will be selected in the individual patient during mono-therapy. The risk is

Table 1. Exceptional resistance phenotypes.

\begin{tabular}{|c|c|}
\hline Gram-negative bacteria & Exceptional phenotypes no. \\
\hline Serratia marcescens and Proteae & Susceptible to colistin \\
\hline Pseudomonas aeruginosa and Acinetobacter spp. & Resistant to colistin \\
\hline Haemophilus influenzae & Resistant to any third-generation cephalosporin, carbapenems, and fluoroquinolones \\
\hline Moraxella catarrhalis & Resistant to ciprofloxacin and any third-generation cephalosporin \\
\hline Neisseria meningitidis & Resistant to any third-generation cephalosporin \\
\hline Neisseria gonorrhoeae & Resistant to third-generation cephalosporin and spectinomycin \\
\hline Gram-positive bacteria & Exceptional phenotypes no. \\
\hline Staphylococcus aureus & $\begin{array}{l}\text { Resistant to vancomycin, teicoplanin, linezolid, quinupristin-dalfopristin, daptomycin, } \\
\text { and tigecycline }\end{array}$ \\
\hline Coagulase-negative staphylococci & Resistant to vancomycin, linezolid, quinupristin-dalfopristin, daptomycin, and tigecycline \\
\hline JK coryneform organisms & $\begin{array}{l}\text { Resistant to vancomycin, teicoplanin, linezolid, quinupristin-dalfopristin, daptomycin, and } \\
\text { tigecycline }\end{array}$ \\
\hline Streptococcus pneumoniae & $\begin{array}{l}\text { Resistant to imipenem, meropenem, vancomycin, teicoplanin, linezolid, quinupristin- } \\
\text { dalfopristin, daptomycin, tigecycline, and rifampicin }\end{array}$ \\
\hline Group A, B, C and G $\beta$-hemolytic streptococci & $\begin{array}{l}\text { Resistant to penicillin, cephalosporins, vancomycin, teicoplanin, linezolid, quinupristin- } \\
\text { dalfopristin, daptomycin, and tigecycline }\end{array}$ \\
\hline Enterococcus spp. & $\begin{array}{l}\text { Resistant to linezolid, daptomycin, and tigecycline. Resistant to teicoplanin but not } \\
\text { vancomycin }\end{array}$ \\
\hline $\begin{array}{l}\text { Enterococcus faecalis, Enterococcus gallinarum, } \\
\text { Enterococcus casseliflavus, } \\
\text { and Enterococcus avium }\end{array}$ & $\begin{array}{l}\text { Susceptible to quinupristin-dalfopristin. Consider likelihood of mis-identification. If also } \\
\text { resistant to ampicillin, it is almost certainly E. faecium }\end{array}$ \\
\hline Enterococcus faecium & $\begin{array}{l}\text { Resistant to quinupristin-dalfopristin. Consider likelihood of mis-identification, especially } \\
\text { if also susceptible to ampicillin }\end{array}$ \\
\hline Anaerobes bacteria & Exceptional phenotypes no. \\
\hline Bacteroides spp. & Resistant to metronidazole and carbapenems \\
\hline Clostridium difficile & Resistant to metronidazole and vancomycin \\
\hline
\end{tabular}

Table 2. Ability to develop resistance to a class of antibiotics for single point mutation.

\begin{tabular}{ll}
\hline Organisms & Antibiotics \\
\hline Staphylococci & Rifampicin, quinolones \\
\hline Streptococcus pneumoniae & Ciprofloxacin \\
\hline Pseudomonas aeruginosa & All antibiotics except colistin \\
\hline Enterobacter, Citrobacter, Serratia, Morganella & Third generation cephalosporin \\
\hline Enterobacteriaceae ESBL & Cefoxitin, cefotetan \\
\hline Enterobacteriaceae & Fosfomycin \\
\hline
\end{tabular}

ESBL, extended-spectrum $\beta$-lactamases. 


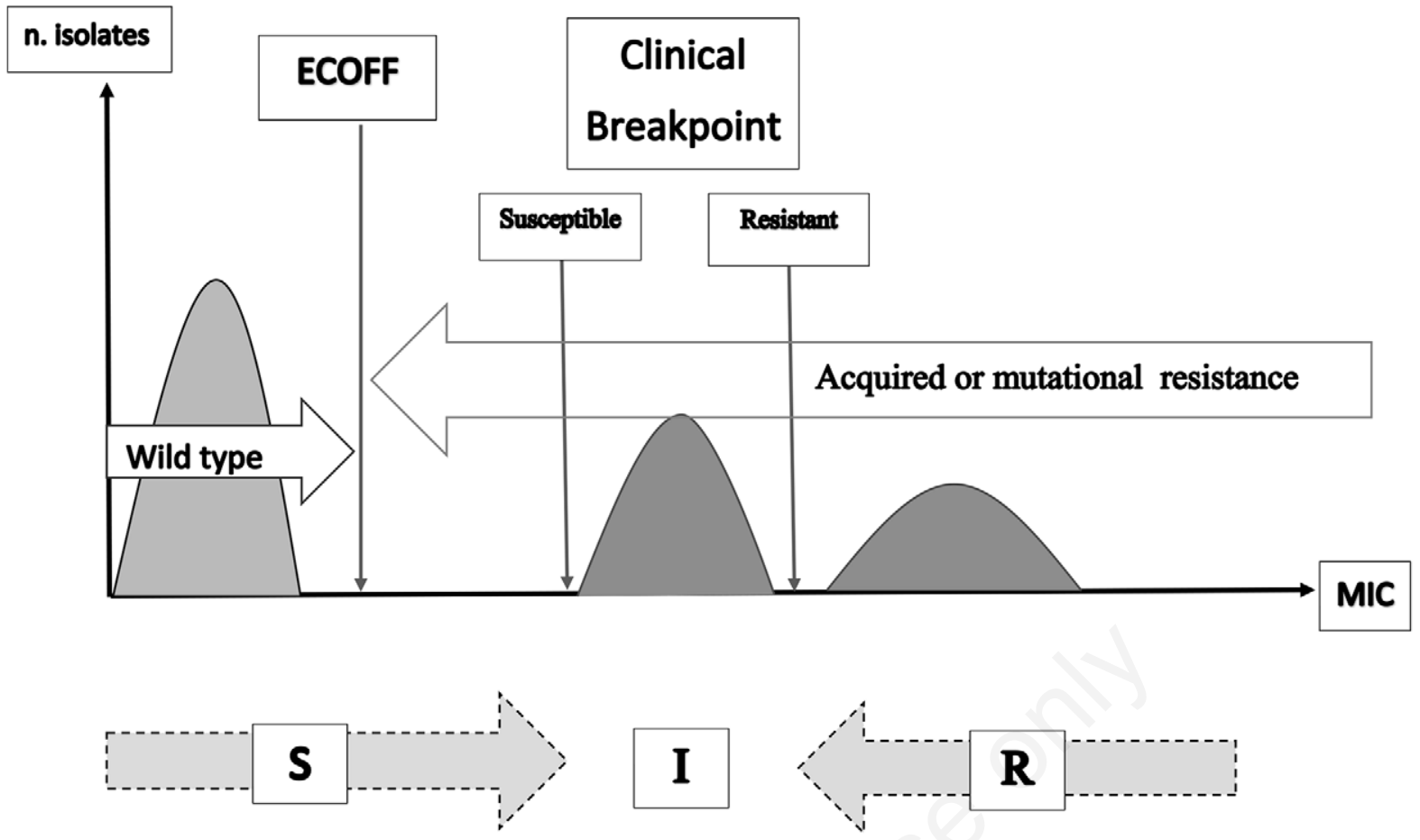

Figure 1. Clinical breakpoints and epidemiological cut-off.

modulated by the site of infection, being increased in those where it is difficult to obtain high drug levels.

\section{Indicator drugs}

An indicator drug is chosen as the member of the drug family to which the mechanism gives the most obvious resistance and it is used to detect the presence of the mechanism that gives resistance not only to the indicator, but also to related agents. Table 3 illustrates the most used indicator drugs. ${ }^{3}$

\section{Key message}

The clinicians should know the criteria adopted by EUCAST for determining the clinical breakpoints.

The expert rules of EUCAST should be shared among clinicians in order to avoid the development of multi-resistant microorganisms.

The phenotypic interpretation of an antibiogram is predictive of the right prescription of an appropriate antibiotic therapy.

MIC breakpoints are developed by employing some combination of: i) MIC frequency distribution analysis; ii) presence or not of known mechanisms of resistance; iii) clinical correlation; iv) evaluation of MICs based on drugs levels in patients (PK/PD).

Clinical breakpoint (Figure 1) indicates likelihood of therapeutic success (susceptible) or failure (resistant) of antimicrobial treatment based on microbiolog- ical findings. ECOFF values separate microorganism without (wild type) and with acquired or mutational resistance.

MIC value should also be assessed in relation to the distance from the breakpoint value of susceptibility.

There is an antibiotic X with a MIC of $0.5 \mathrm{mg} / \mathrm{L}$ and breakpoint $2 \mathrm{mg} / \mathrm{L}$, and an antibiotic $\mathrm{Y}$ with a MIC of $2 \mathrm{mg} / \mathrm{L}$ but breakpoint of $16 \mathrm{mg} / \mathrm{L}$ : the drug with a MIC more favorable is the antibiotic Y (Figure 2).

The expert rules dictated by EUCAST, which are
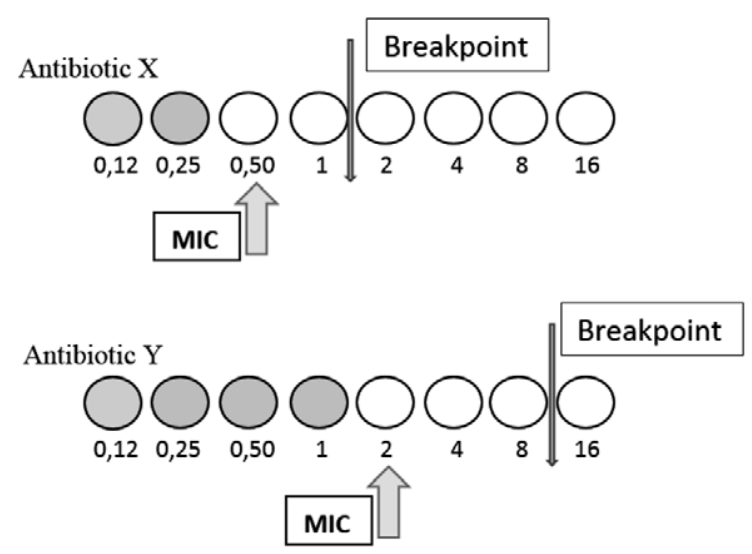

Figure 2. The drug with a MIC more favorable is the antibiotic Y. 
Table 3. Useful indicator antibiotic drugs and interpretative rules.

\begin{tabular}{lll}
\hline Organism & Agents tested & Action or inference \\
\hline Staphylococcus spp. & Oxacillin or methicillin, cefoxitin & Resistance to all $\beta$-lactam except ceftobiprole and ceftaroline \\
\hline Staphylococcus spp. & Benzylpenicillins & $\begin{array}{l}\text { Resistance to penicillins apart from isoxazolyl-penicillins and combination } \\
\text { with } \beta \text {-lactamase inhibitors }\end{array}$ \\
\hline Staphylococcus spp. & Erythromycin & Inducible clindamycin resistance likely \\
\hline Staphylococcus spp. & Erythromycin and clindamycin & $\begin{array}{l}\text { If resistance to macrolides but susceptible to clindamycin: test for constitutive } \\
\text { MLS }_{\text {B }} \text { resistance. Quinuprostin/dalfopristin likely to be bacteriostatic, not } \\
\text { bactericidal }\end{array}$
\end{tabular}

Staphylococcus spp. Gentamicin

Resistance to gentamicin is generally caused by a production of enzyme that determines loss of synergism of all aminoglycosides (except streptomycin) with $\beta$-lactam agents and glycopeptides irrespective of MIC value

Staphylococcus spp. Ciprofloxacin or ofloxacin

Acquisition of at least one target mutation to resistance to all fluoroquinolones. First-step mutation may lead to resistance development under therapy with other quinolones

\begin{tabular}{ll}
\hline Staphylococcus spp. & Levofloxacin and \\
\hline Streptococcus pneumoniae & Oxacillin \\
\hline $\begin{array}{ll}\text { Viridans groups } \\
\text { of streptococci }\end{array}$ & Benzylpenicillin \\
\hline
\end{tabular}

Acquisition of combined mutation that leads to complete or partial cross-resistance to all fluoroquinolones

Streptococcus spp. Erythromycin and clindamycin

If resistant by screening, determine MIC of benzylpenicillins and other $\beta$-lactam agents and report as interpreted for each of the drug: production of mosaic leads to various patterns of $\beta$-lactam resistance

If resistant by screening, determine MIC of amoxicillin or ampicillin and cefotaxime or ceftriaxone and report as interpreted for each of the drug: production of mosaic leads to various patterns of $\beta$-lactam resistance

Streptococcus pneumoniae Ciprofloxacin or ofloxacin
If resistance to erythromycin but susceptible to clindamycin then test for inducible $\mathrm{MLS}_{\mathrm{B}}$ resistance. Streptococci may be resistant to macrolides by production of $\mathrm{MLS}_{\mathrm{B}}$ phenotype or by production of an efflux pump

Streptococcus pneumoniae Ciprofloxacin or ofloxacin

Acquisition of at least one target mutation to resistance to all fluoroquinolones. First-step mutation may lead to resistance development under therapy with other quinolones. First step mutation can be reliably detected in test with norfloxacin

\begin{tabular}{|c|c|c|}
\hline Streptococcus pneumoniae & Levofloxacin and moxifloxacin & $\begin{array}{l}\text { Acquisition of combined mutation that leads to complete or partial } \\
\text { cross-resistance to all fluoroquinolones }\end{array}$ \\
\hline Enterococcus spp. & Ampicillin & Resistance to ureidopenicillins and carbapenems \\
\hline E. faecalis & Ampicillin & $\begin{array}{l}\text { It is probably } E \text {. faecium, but may be less frequent species or may have } \\
\text { acquired resistance: check speciation }\end{array}$ \\
\hline Enterococcus spp. & Gentamicin & $\begin{array}{l}\text { Resistance to gentamicin is generally caused by a production of enzyme that } \\
\text { determines loss of synergism of all aminoglycosides (except streptomycin) } \\
\text { with } \beta \text {-lactam agents and glycopeptides irrespective of MIC value }\end{array}$ \\
\hline Enterobacteriaceae & $\begin{array}{l}\text { Cefotaxime, ceftriaxone, } \\
\text { ceftazidime, cefepime, } \\
\text { amoxicillin-clavulanate, } \\
\text { ampicillin-sulbactam and } \\
\text { piperacillin-tazobactam }\end{array}$ & $\begin{array}{l}\text { If intermediate or resistant to any third generation or fourth generation } \\
\text { oxyimino-cephalosporin and susceptible to combination of penicillin and } \\
\text { a } \beta \text {-lactamase inhibitor they are often ESBL; then report as tested }\end{array}$ \\
\hline $\begin{array}{l}\text { Enterobacter spp. } \\
\text { Citrobacter freundii } \\
\text { Serratia } \text { spp. } \\
\text { Morganella Morganii }\end{array}$ & $\begin{array}{l}\text { Cefotaxime, ceftriaxone } \\
\text { and ceftazidime }\end{array}$ & $\begin{array}{l}\text { Selection of AmpC-derepressed cephalosporin-resistant mutants may occur } \\
\text { during therapy. Third-generation cephalosporin in combination with } \\
\text { quinolones has may also lead selection of resistant mutant. The selection } \\
\text { risk is diminished for cefepime and cefpirome }\end{array}$ \\
\hline $\begin{array}{l}\text { Enterobacteriaceae } \\
\text { (mostly Klebsiella spp. and } \\
\text { Escherichia coli) }\end{array}$ & Ticarcilin, piperacillin & $\begin{array}{l}\text { Ticarcillin-hydrolyzing } \beta \text {-lactamases also attack piperacillin, but resistance } \\
\text { expression may be low-level }\end{array}$ \\
\hline Enterobacteriaeceae & Ciprofloxacin & Resistant to all fluoroquinolones \\
\hline Klebsiella/E. coli & Ceftazidime & $\begin{array}{l}\text { Likely ESBL producer. Resistant to all cephalosporin except cephamycins } \\
\text { (cefoxitin, cefotetan) }\end{array}$ \\
\hline
\end{tabular}

$\mathrm{MLS}_{\mathrm{B}}$, macrolide-lincosamide-streptogramin B; MIC, minimum inhibitory concentration; ESBL, extended-spectrum $\beta$-lactamases. 
based on current clinical breakpoints and knowledge of resistance mechanisms, describe the actions to be taken on the basis of specific results with the aim to help the clinician, and the microbiologist, in an interpretative reading of an antibiogram. ${ }^{1,3}$

\section{Part II: Microorganisms}

\section{Staphylococcus aureus}

Staphilococcus aureus has developed many mechanisms of resistance: to penicillins through penicillinases and then also to the penicillinase-resistant penicillins such as oxacillin and methicillin by penicillin-binding protein (PBP) modification.

Production of penicillinase in staphylococci is very common ( $>90 \%)$ and it leads to phenotypic resistance to all penicillins except the isoxazolyl analogues.

Staphylococci can be resistant to the isoxazolylpenicillins when an abnormal PBP $\left(\mathrm{PBP}_{2 \mathrm{a}}\right.$ encoded by mecA gene) lead to cross-resistance to all $\beta$-lactams, except new anti-methicillin-resistant $S$. aureus (MRSA) cephalosporins. MecA-mediated resistance is commonly referred to as methicillin (or oxacillin) resistance. All staphylococci resistant to methicillin, oxacillin, and/or cefoxitin, should be considered as resistant to all $\beta$-lactams, except ceftaroline and ceftobiprole. ${ }^{4}$ Recently a mecC-mediated resistance was described.

Detection of oxacillin-resistance in $S$. aureus (ORSA) isolates is mandatory: ${ }^{5}$ the prevalence of MRSA/ORSA in Italy has been constant for several years and about $40 \%$ of all isolates.

The determination of sensitivity to methicillin may be difficult, since the mechanism of resistance may be heterogeneous (namely there is a limited number of colonies that are difficult to detect in vitro but that cause treatment failure during a therapy with oxacillin).

To better detect the methicillin resistance of $S$. aureus some measures have been suggested, as the use of screening agar with addition of $4 \% \mathrm{NaCl}$, incubated at $35^{\circ} \mathrm{C}$, and addition of oxacillin $(6 \mathrm{mg} / \mathrm{L})$ : the growth of a single colony is sufficient to define a MRSA strain. With regard to the Kirby-Bauer test, it is however recommended to use disks containing cefoxitin $(30 \mu \mathrm{g})$, this test has the same sensitivity and specificity of screening with oxacillin. According to the EUCAST criteria, it is used only the cefoxitin disk, but as regards the comparison of MIC, it is recommended to use only oxacillin (and not cefoxitin). ${ }^{1,2}$

A small proportion of $S$. aureus may present at various degrees of resistance to glycopeptides [vancomycin-intermediate $S$. aureus (VISA), vancomycinresistant $S$. aureus (VRSA)].

VISA are $S$. aureus with a vancomycin MIC between 4 and $8 \mathrm{mg} / \mathrm{L}$; rarely some strains may have vancomycin MIC $\geq 16 \mathrm{mg} / \mathrm{L}$ (VRSA, very rare in Europe). These previous breakpoints were identified on the basis of clinical studies that have demonstrated the failure of therapy with vancomycin during bacteremia caused by $S$. aureus with vancomycin MIC of 4 $\mathrm{mg} / \mathrm{L} .{ }^{6}$ EUCAST has changed the $\mathrm{BC}$ to $2 \mathrm{mg} / \mathrm{L}$ for vancomycin and teicoplanin, respectively; therefore, strains with MIC $>2 \mathrm{mg} / \mathrm{L}$ are at present clinically resistant to glycopeptides, but definition of VISA actually remains for describing non-VanA mediated vancomycin resistance and for distinguishing it from vanA mediated one. VISA are strains with thicker cellwall reducing the activity of cell-wall agents such as glycopeptides.

VRSA strains have acquired the vancomycin resistance gene vanA from vancomycin resistant Enterococcus with a completely change in the lateral chain of cell-wall with a depsipeptide instead of dipeptide, therefore glycopeptides are completely inactive against these strains. $^{\text {? }}$

The identification of VISA strains can be difficult because they grow more slowly than other $S$. aureus: susceptibility testing should not be read prior to $24 \mathrm{~h}$ of incubation and the Kirby-Bauer test may prove as inadequate as some automated methods. ${ }^{8,9}$ Therefore, the laboratories which use these methods should test the sensitivity of S.aureus by screening the method of agar with brain-heart infusion with $6 \mathrm{mg} / \mathrm{L}$ of vancomycin and bacterial inoculum equal to $10^{6} \mathrm{CFU}$.

The definition of hetero-VISA (hVISA) is a strain of $S$. aureus that have MIC for vancomycin in the range of 1-2 $\mathrm{mg} / \mathrm{L}$, which is in the range of susceptibility. Among this population, there are strains every $10^{6}$ colonies with vancomycin MIC of 4 or $8 \mathrm{mg} / \mathrm{L}$ or even higher.

The identification of hVISA is even more difficult and it is recommended the use of a screening test on Muller-Hinton agar with addition of teicoplanin 5 $\mathrm{mg} / \mathrm{L}$, greater inoculum and greater incubation time. The growth of a single colony is used to identify the strain as hVISA. ${ }^{10}$ Therefore, clinicians should suspect h-VISA strains in case of $S$. aureus with vancomycin MIC 1 or 2 that does not respond to a mono-therapy with a glycopeptide.

Although the macrolides, lincosamides and streptogramins have different chemical structures, they share similar mechanisms of action, and can be affected by the same resistance mechanisms. Erythromycin is considered to be the class representative for clarithromycin and azithromycin, resistance can occur with or without cross-resistance to clindamycin and lincosamides. Resistance to these compounds is generally encoded by erm genes that confer constitutive or inducible macrolide-lincosamide-streptogramin $B\left(M_{B} S_{B}\right)$ phenotypes or by the production of an efflux pump (M-phenotype, conferring resistance to erythromycin but not 
to clindamycin and/or streptogramins). ${ }^{11}$ The recommended disk diffusion test for inducible $\mathrm{MLS}_{\mathrm{B}}$ resistance consists of an erythromycin disk in close proximity to a clindamycin disk. If the inhibition halo around clindamycin disk is flattened towards the disk of erythromycin, this means that an inducible mechanism of resistance is present and therefore the clinical use of clindamycin should be discouraged. ${ }^{3}$ For staphylococcal isolates that are simultaneously resistant to erythromycin and clindamycin or lincomycin, a warning of reduced susceptibility to the combination quinupristin-dalfopristin and loss of bactericidal activity should be included in the susceptibility test report. ${ }^{12,13}$

\section{Key message}

- In S. aureus methicillin-resistant screening is mandatory.

- Methicillin-resistance should be read as oxacillinresistance (MRSA=ORSA).

- Glycopeptide MIC is important to predict the possible presence of hVISA isolates that may fail monotherapy with a glycopeptide.

\section{Enterococci}

Enterococci are opportunistic pathogens, multi-resistant, often isolated from patients treated with several antibiotics and hospitalized for long time. Often they cause urinary infection and endocarditis, and Enterococci have several mechanisms of resistance.

All Enterococci are considered to be intrinsically resistant to cephalosporins (Appendix).

They are also intrinsically resistant to anti-staphylococcal penicillins, clindamycin, aminoglycosides at low concentrations and, in vivo, to trimethoprim. Mono-therapy with ampicillin, penicillin or glycopeptide is effective except in endocarditis. Mono-therapy is never bactericidal.

In cases of endocarditis it is mandatory to test for the detection of penicillinase with the colorimetric test or using a higher bacterial inoculation. Detection of this resistance is important because these strains, although resistant to ampicillin and penicillin, remain susceptible to the associations between penicillin and suicide inhibitors of penicillinase.

Enterococci, in particular E. faecium, have often resistance to glycopeptides, mediated by plasmids and transposons. In this case the phenotype VanA confers resistance to vancomycin and teicoplanin, respectively. Resistance to ampicillin is almost always present in $E$. faecium. ${ }^{14}$ Instead E. faecalis is susceptible to ampicillin, and resistant strains are due to $\beta$-lactamase production. Therefore, a strain of E. faecium susceptible to ampicillin is an impossible phenotype and the species has to be rechecked. Ampicillin susceptibility allows to infer susceptibility to amoxicillin, piperacillin and imipenem (but not to meropenem, because genetic resistance).
It is important to know that the optimal therapy for severe enterococci infections provides association between a penicillin or a glycopeptide and an aminoglycoside, this being a synergistic and bactericidal combination of drugs. Drugs that destroy the bacterium cell wall allow the entry of a massive amount of aminoglycosides. In case of high-level resistance to aminoglycosides, combination does not work, because of modification of the antibiotic inside the microorganism or because of a change of the target enzyme. Therefore, in the lab, this kind of resistance has to be performed, using high concentration of gentamicin $(>128 \mathrm{mg} / \mathrm{L})$ or streptomycin $(>1000 \mathrm{mg} / \mathrm{L})$. Streptomycin is tested by itself because it has different resistance mechanisms.

EUCAST recommended that report as resistant enterococci to all aminoglycosides, except streptomycin, if they are resistant to high levels gentamicin. Highlevel aminoglycosides resistant Enterococcus faecalis (HLAR) may be treated with ampicillin associated with ceftriaxone, because there is evidence of sinergistic in vivo activity in endocarditis.

EUCAST did not recommend testing quinolones because they were considered as ineffective, except that in uncomplicated urinary tract infections.

\section{Key message}

High-level resistance to aminoglycosides is very important to predict the efficacy of the combination between penicillins or glycopeptides and aminoglycosides.

\section{Streptococcus pneumoniae}

Streptococcus pneumoniae is the leading cause of pneumonia, meningitis and otitis in many countries. Resistance to $\beta$-lactams in S. pneumoniae is increasing in some countries, owing to the production of mosaic PBPs that lead to various patterns of $\beta$-lactam resistance. The oxacillin disk is traditionally used in screening tests to indicate benzylpenicillin susceptibility, but when clinically needed and the isolated is interpreted as indicating benzylpenicillin-resistant, MICs of cephalosporin and carbapenems should be determined.

EUCAST, in meningitis, defined as only sensitive those strains with MIC $\leq 0.06 \mathrm{mg} / \mathrm{L}$.

The strains with high resistance to penicillin may also have resistance to ceftriaxone and to carbapenems. ${ }^{15}$

As for staphylococci, macrolide resistance should be tested, it can be inducible by erm $\mathrm{B}$ gene that also mediates resistance to clindamycin and streptogramin B.

Resistance to quinolones in $S$. pneumoniae is worry because it may be selected, during treatment, only a point mutation in the target.

EUCAST recommended to use as screening the norfloxacin disk: if inhibition zone is $>12 \mathrm{~mm}$ it can be reported as susceptible to levofloxacin and moxifloxacin and intermediate to ciprofloxacin. Strains resistant to screening test should be tested for individual 
drugs. If pneumococcus is resistant to ciprofloxacin and susceptible to levofloxacin and moxifloxacin we need to know that the strain has already acquired a first mutation of resistance in the target gene. If it is resistant to levofloxacin and moxifloxacin it cannot be susceptible to other quinolones.

EUCAST introduced breakpoints for ciprofloxacin susceptibility, but the limit is so low (MIC $\leq 0.12 \mathrm{mg} / \mathrm{L}$ ) that the wild type strains are considered always intermediate. The levofloxacin breakpoint was set at $2 \mathrm{mg} / \mathrm{L}$ because of the distribution of wild type strains, in this case the $\mathrm{BC}$ might be too high facilitating the selection of resistance during mono-therapy in clinical practice.

\section{Key message}

In S. pneumoniae penicillin resistance is particularly important in strains isolates in meningitis.

\section{Enterobacteriaceae}

In the family Enterobacteriaceae there are many genres: Escherichia, Klebsiella spp., Enterobacter spp., Proteus spp., Shigella spp., Salmonella spp., Citrobacter spp. and others. They are especially germs from gut and often cause severe endogenous infections. The massive and indiscriminate use of antibiotics has led to the diffusion of important resistances.

Interpretive reading of the antibiogram is commonly based on $\beta$-lactams in Gram-negative bacilli. ${ }^{16}$ Some authors claimed that MIC breakpoints set at appropriate levels (decreasing their values) can detect the presence of clinically significant resistance mechanisms. ${ }^{17}$

The main mechanism of resistance in Enterobacteriaceae is due to the production of $\beta$-lactamases.

Enterobacteriaceae $\beta$-lactamases (but this is also true for P. aeruginosa and Acinetobacter spp.) are divided into 4 classes of Ambler. According to the Ambler molecular classification of these enzymes they can be grouped, based on the primary structure, into 4 classes (A-D) genetically different.

First $\beta$-lactamases, which appeared in the ' 60 s, are penicillinases (TEM-1, TEM-2, SHV-1), which are inhibited by suicide penicillinase inhibitors such as sulbactam, tazobactam, clavulanic acid.

TEM penicillinases are part of the class A and are found mainly in gram negative bacteria and are responsible for resistance to penicillins and broad spectrum cephalosporins. The variants of the TEM gene are caused by point mutations.

Another important penicillinase is $S H V$, which belongs to the class A of $\beta$-lactamase. The gene encoding this $\beta$-lactamase may be present on either plasmids or on the chromosome of the bacterium and is frequently found in strains of $K$. pneumoniae.

Third and fourth generation cephalosporins have been developed to overcome these broad-spectrum penicillinases, but through point mutations they have developed ESBL. ESBLs are enzymes that hydrolyze most penicillins and cephalosporins, including oxyimino- $\beta$-lactam compounds (cefuroxime, third- and fourth-generation cephalosporins and aztreonam) but not cephamycins or carbapenems. ESBLs in vitro are inhibited by penicillinases suicide inhibitors.

The recommended strategy for the detection of ESBLs in Enterobacteriaceae is based on non-susceptibility to indicator oxymino-cephalosporins, followed by phenotypic confirmation tests. Cefpodoxime is the most sensitive individual indicator cephalosporin for ESBL production detection, but is less specific than the combination of cefotaxime (or ceftriaxone) and ceftazidime. ${ }^{18,19}$

Clinicians should know the characteristics of these enzymes because the recognition of ESBL in the laboratory can be difficult and nowadays it is not indicated anymore.

In vitro detection of ESBLs is difficult because not all enzymes inactivate in the same way substrate drugs: some enzymes hydrolyze more ceftazidime, others more cefotaxime. So, both drugs should be used in the susceptibility tests. There are many types of different enzymes among ESBLs, some of which have evolved through mutations starting from the penicillinase while others have arisen as new enzymes (for example, CTX-M). CTX-M has spread epidemically and it is capable of hydrolyzing better cefotaxime than ceftazidime. Some CTX-M are also active against cefepime, but they are all susceptible to acid clavulanic.

In addition, for ESBLs exists the inoculum effect (at least in vitro), for which the resistance should be noted only with larger amounts than those normally used. The inoculum effect was particularly evident for piperacillin/tazobactam and cefepime. In brief, we could have a susceptible in vitro result that does not correspond with a high probability of success in vivo.

If ESBL production is suspected, according to the new EUCAST parameters, it should be made a confirmatory test, which relies on the use in combination of cephalosporins and clavulanic acid: the test result is positive when with combination drugs MICs decreases by at least 8 times, or there is an increase of inhibition halo.

With the new EUCAST rules for Enterobacteriaceae, third-generation and fourth-generation cephalosporins MICs should be reported as found, and the old expert rule recommending modification in resistant of reporting category for ESBL producers is no longer appropriate. This recommendation, which also applies to plasmid-mediated $\mathrm{AmpC}$ producers, is now included in the EUCAST breakpoint tables.

AmpC is another type of $\beta$-lactamase and represents a heterogeneous group of genes that may be located on chromosome or plasmids. Many strains 
belonging to the Enterobacteriaceae family have one or more $\mathrm{AmpC}$ genes, which, however, are expressed mainly in strains of Enterobacter spp. (on chromosome) and Proteus spp. (on plasmid). Belonging to the class $\mathrm{C}$, the encoded enzyme can cause resistance to penicillins and cephalosporins. The peculiarity, which then makes identification difficult, is that it is not inhibited by clavulanic acid and tazobactam, in contrast to ESBL. The AmpC have spectrum of action similar to that of ESBLs and must be recognized for a proper therapy. They are resistant to all suicide inhibitors and also to cephamycins making the germ resistant to cefoxitin and cefotetan.

Clavulanic acid resistance does not allow using the screening test used for ESBLs. So AmpC may be assumed if we observe resistance to cephalosporins associated with resistance to suicide inhibitors and cefoxitin, or if there is even residual susceptibility to cefepime.

We might recognize the phenotype of an AmpC producer in the most probable species such as Enterobacter spp. and Proteae, if the isolate is resistant to some third-generation cephalosporins and cefoxitin. For this reason, it was important that antibiograms reported cefoxitin result.

Trying to synthesize ESBL and AmpC in Enterobacteriaceae: ceftazidime is the best indicator for ESBL, TEM and SHV, while cefotaxime is the best indicator for CTX-M. The resistance to ceftazidime and/or cefotaxime in the absence of resistance to cefoxitin strongly indicates the presence of ESBL, while the resistance to cefoxitin is strongly indicative of AmpC production.

All confirmed ESBL-positive organisms have been considered in the past resistant to cephalosporins and practically only susceptible to carbapenems, and this fact has brought to an overuse of carbapenems with selection of carbapenemase producers Gram-negative rods. The spread of Gramnegative multi-drug resistant (MDR) has become an emergency worldwide in the last decades. Another gene that confers resistance to $\beta$-lactams belongs to the type OXA (group D). Unlike TEM and SHV, OXA gives the bacteria cephalotin and ampicillin resistance, and due to its strong hydrolytic activity is also able to hydrolyze oxacillin. This $\beta$-lactamase is detected in strains of $E$. coli and $K$. pneumoniae but most of these enzymes were found in Acinetobacter baumannii. The OXA-penicillinase type belongs to the group D of $\beta$-lactamase. Carbapenemase-producing strains are characterized by their resistance to virtually all $\beta$-lactam antibiotics, including the cephalosporins and carbapenems, and often to fluoroquinolones, aminoglycosides and cotrimoxazole.

Three types of carbapenemases are now commonly identified in Enterobacteriaceae. They are the Ambler class A (serine carbapenemase) of the car- bapenemases (KPC) type, class B (metallo-carbapenemase), and class D (OXA carbapenemase).

Recent data provide evidence to support that carbapenems may be still active in KPC producers $K$. pneumoniae..$^{20,21}$ This is why KPC is a weak carbapenemase, so other mechanism of resistant such as porin deficiency or activated efflux pump are necessary to increase the MICs for carbapenems to resistance levels. Therefore, detection of carbapenemases in clinical microbiology labs is a challenging issue. Adequate detection of carbapenemase-producing microorganism in the routine diagnostic laboratory is essential for the correct choice of antibiotic therapy and to undertake appropriate hospital hygiene precautions.

The first step in the screening of carbapenemase producers is to compare the results of susceptibility testing with the breakpoint values of carbapenems, usually using automatic systems, but more accurately with broth microdilution methodology or E-test.

Carbapenemases can have variable effects on carbapenems, ${ }^{22,23}$ therefore epidemiological breakpoint should be used to correctly identify these strains with confirmatory tests. Combined resistance mechanisms may also affect carbapenem susceptibility (e.g., combination of derepressed AmpC or ESBL and decreased permeability). ${ }^{24}$ Ertapenem is less specific because isolates with AmpC/ESBL and decreased permeability to porin have higher MICs for ertapenem than for imipenem or meropenem..$^{25}$ Meropenem at a cut-off $>0.12 \mathrm{mg} / \mathrm{L}$ has the best balance of sensitivity and specificity for suspecting carbapenemase-producing Enterobacteriaceae. $^{3}$

Special attention should be paid to reduced susceptibility to carbapenems that may be related to true carbapenemases, not only for producers of class B or class A KPC, but also for those expressing OXA-48, a class D carbapenemase that is increasingly being identified in Enterobacteriaceae. ${ }^{26}$

Phenotypic confirmation of carbapenemase production is based on detection of a diffusible carbapenemase (the modified Hodge Test) and in vitro inhibition of enzyme activity upon addiction of a phenotypic inhibitor (carbabenemase inhibition tests). ${ }^{27}$ The modified Hodge test is a phenotypic screening test for carbapenemases that is used for epidemiological purposes, and its use is currently proposed by CLSI, unfortunately is less susceptible and not used anymore. Carbapenem screening breakpoint, clinical breakpoint and epidemiological cut/off value for $E n$ terobacteriaceae are shown in Table 4, while phenotypic methods are summarized in the Table 5.

\section{Key message}

- ESBL and AmpC in Enterobacteriaceae: ceftazidime is the best indicator for ESBL, TEM and SHV, while cefotaxime is the best indicator for CTX-M. 
Table 4. Carbapenem screening breakpoint, clinical breakpoint and epidemiological cut/off value (mg/L).

\begin{tabular}{|c|c|c|c|c|c|}
\hline & $\begin{array}{c}\text { Meropenem } \\
\text { Escherichia coli } \\
\text { Klebsiella pneumoniae }\end{array}$ & $\begin{array}{l}\text { Meropenem } \\
\text { Proteus spp. }\end{array}$ & $\begin{array}{c}\text { Imipenem } \\
\text { Escherichia coli }\end{array}$ & $\begin{array}{c}\text { Imipenem } \\
\text { Klebsiella pneumoniae }\end{array}$ & $\begin{array}{c}\text { Ertapenem } \\
\text { allenterobacteria }\end{array}$ \\
\hline $\begin{array}{l}\text { Carbapenem screening } \\
\text { breakpoint }\end{array}$ & $\geq 0.5$ & $\geq 0.5$ & $\geq 2$ & $\geq 2$ & $\geq 0.5$ \\
\hline Epidemiological cut-off value & $\mathrm{S} \leq 0.125$ & $\mathrm{~S} \leq 0.25$ & $\mathrm{~S} \leq 0.5$ & $\mathrm{~S} \leq 1$ & $\mathrm{~S} \leq 0.064$ \\
\hline EUCAST BC & $\mathrm{S} \leq 2$ & $\mathrm{~S} \leq 2$ & $\mathrm{~S} \leq 2$ & $\mathrm{~S} \leq 2$ & $\mathrm{~S} \leq 0.5$ \\
\hline CLSI BC & $\mathrm{S} \leq 1$ & $\mathrm{~S} \leq 1$ & $\mathrm{~S} \leq 1$ & $\mathrm{~S} \leq 1$ & $\mathrm{~S} \leq 1$ \\
\hline
\end{tabular}

EUCAST, European Committee of Antimicrobial Susceptibility Testing; BC, clinical breakpoint; CLSI, Clinical and Laboratory Standards Institute.

Table 5. Interpretation scheme of phenotypic carbapenemase confirmation tests.

\begin{tabular}{lccccc}
\hline Confirmation test & $\begin{array}{c}\text { Carbapenemase } \\
\text { Class A }\end{array}$ & $\begin{array}{c}\text { Carbapenemase } \\
\text { Class B }\end{array}$ & $\begin{array}{c}\text { Carbapenemase } \\
\text { Class D* }\end{array}$ & $\begin{array}{c}\text { AmpC + reduced } \\
\text { permeability }\end{array}$ & $\begin{array}{c}\text { ESBL + reduced } \\
\text { permeability }\end{array}$ \\
\hline Hodge test & + & + & + & - & + \\
\hline Meropenem \pm boronic acid & + & - & - & + & + \\
\hline Meropenem \pm cloxacillin & - & - & - & + & - \\
\hline Meropenem \pm EDTA & - & + & - & -
\end{tabular}

*In case of all test negative, except Hodge test, it may be useful to test temocillin; if minimum inhibitory concentration (MIC) is $>128 \mathrm{mg} / \mathrm{L}, \mathrm{Oxa}-48$ phenotype might be possible. ESBL, extended-spectrum $\beta$-lactamases; EDTA, ethylenediaminetetraacetic acid.

- The resistance to ceftazidime and/or cefotaxime in the absence of resistance to cefoxitin strongly indicates the presence of ESBL, while the resistance to cefoxitin is strongly indicative of AmpC production.

- Carbapenemases should be suspected when the MIC is in the range of MICs between the CB and the epidemiological breakpoint. In this case a phenotypic confirmatory test should be performed.

\section{Conclusions}

In internal medical wards, especially in Italy, where there are many outbreak of MDR microorganisms, a physician should be able to recognize the phenotype of resistance of the most important pathogens.

MRSA should be identified correctly and treated properly. In case of bacteremia caused by enterococci a clinician should be able to choose the bactericidal combination aimed to solve the infections.

For enterobacteria, the clinical skills of recognition of ESBL, AmpC and KPC expression, should be mandatory, especially because we are facing a dramatic outbreak of these resistance phenotypes, therefore the right recognition would be able to treat properly and to develop isolation strategy aimed to reduce the diffusion of these pathogens inside the hospitals.

\section{References}

1. European Committee for Antimicrobial Susceptibility Testing (EUCAST). Breakpoint Table for interpretation of MICs and zone diameters. Version 6.0. 2016. Available from: http://www.eucast.org

2. Clinical and Laboratory Standards Institute (CLSI). Performance standards for antimicrobial susceptibility testing: nioneteenth informational supplement. Document M-100-S19. Wayne, PA: CLSI; 2010.

3. Leclercq R, Cantón R, Brown DF, et al EUCAST expert rules in antimicrobial susceptibility testing. Clin Microbiol Infect 2011;19:141-60.

4. Chambers HF, Sachdeva M. Binding of beta-lactam antibiotics to penicillin-binding proteins in methicillin-resistant Staphylococcus aureus. J Infect Dis 1990;161: 1170-6.

5. Brown DF. Detection of methicillin/oxacillin resistance in staphylococci. J Antimicrob Chemother 2001;48:65-70.

6. Tenover FC, Mollering RC. The rationale for revising the clinical and laboratory standards institute vancomycin minimal inhibitory concentration interpretative criteria for Staphylococcus aureus. Clin Infect Dis 2007;44:1208-15.

7. Chang S, Sievert DM, Hageman JC, et al. Infection with vancomycin-resistant Staphylococcus aureus containing the vanA resistance gene. N Engl J Med 2003;348:1342-7.

8. Tenover FC, Lancaster MV, Hill BC, et al. Characterization of staphylococci with reduced susceptibility to vancomycin and other glycopeptides. J Clin Microbiol 1998;36:1020-7.

9. Tenover FC, Weigel LM, Appelbaum PC, et al. Vancomycin-resistant Staphylococcus aureus isolate from a 
patient in Pennsylvania. Antimicrobial Agents Chemother 2004:48:275-80.

10. Wootton M, MacGowan AP, Walsh TR, Howe RA. A multicenter study evaluating the current strategies for isolating Staphylococcus aureus strains with reduced susceptibility to glycopeptides. J Clin Microbiol 2007; 45:329-32.

11. Leclercq R. Mechanisms of resistance to macrolides and lincosamides: nature of the resistance elements and their clinical implications. Clin Infect Dis 2002;34:482-92.

12. Entenza JM, Drugeon H, Glauser MP, Moreillon P. Treatment of experimental endocarditis due to erythromycin-susceptible or -resistant methicillin-resistant Staphylococcus aureus with RP 59500. Antimicrob Agents Chemother 1995;39:1419-24.

13. Batard E, Jacqueline C, Boutoille D, et al. Combination of quinupristin-dalfopristin and gentamicin against methicillin-resistant Staphylococcus aureus: experimental rabbit endocarditis study. Antimicrob Agents Chemother 2002;46:2174-8.

14. Fontana R, Ligozzi M, Pittaluga F, Satta G. Intrinsic penicillin resistance in enterococci. Microb Drug Resist 1996;2:209-13.

15. Low DE. Changing trends in antimicrobial-resistant pneumococci: it's not all bad news. Clin Infect Dis 2005;41:S228-33.

16. Livermore DM. $\beta$-lactamases in laboratory and clinical resistance. Clin Microbiol Rev 1995;8:557-84.

17. Kahlmeter G. Breakpoints for intravenously used cephalosporins in Enterobacteriaceae-EUCAST and CLSI breakpoints. Clin Microbiol Infect 2008;14: 169-74.

18. Hope R, Potz NA, Warner M, et al. Efficacy of practiced screening methods for detection of cephalosporin-resis- tant Enterobacteriaceae. J Antimicrob Chemother 2007; 59:110-3

19. Oliver A, Weigel LM, Rasheed JK, et al. Mechanisms of decreased susceptibility to cefpodoxime in Escherichia coli. Antimicrob Agents Chemother 2002;46:3829-36.

20. Daikos GL, Petrikkos P, Psichogiou M, et al. Prospective observational study of the impact of VIM-1 metallo- $\beta$-lactamase on the outcome of patients with Klebsiella pneumoniae bloodstream infections. Antimicrob Agents Chemother 2009;53:1868-73.

21. Daikos GL, Markogiannakis A. Carbapenemase-producing Klebsiella pneumoniae: (when) might we still consider treating with carbapenems? Clin Microbiol Infect 2011;17:1135-41.

22. Walsh TR, Toleman MA, Poirel L, Nordmann P. Metallo-b-lactamases: the quiet before the storm? Clin Microbiol Rev 2005;18:306-25.

23. Poirel L, Pitout JD, Nordmann P. Carbapenemases: molecular diversity and clinical consequences. Future Microbiol 2007;2:501-12.

24. Cohen Stuart J, Leverstein-Van Hall MA; Dutch Working Party on the Detection of Highly Resistant Microorganisms. Guideline for phenotypic screening and confirmation of carbapenemases in Enterobacteriaceae. Int J Antimicrob Agents 2010;36:205-10.

25. Woodford N, Dallow J, Hill RLR, et al. Mechanisms of ertapenem resistance among Klebsiella and Enterobacter submitted in the United Kingdom to a reference laboratory. Int J Antimicrob Agents 2007;29:456-9.

26. Livermore DM. Has the era of untreatable infections arrived? J Antimicrob Chemother 2009;64:i29-36.

27. Miriagou V, Cornaglia G, Edelstein M, et al. Acquired carbapenemases in Gram-negative bacterial pathogens: detection and surveillance issue. Clin Microbiol Infect 2010;16:112-22. 